\title{
Inhibition of MDM2 via Nutlin-3A: A Potential Therapeutic Approach for Pleural Mesotheliomas with MDM2-Induced Inactivation of Wild-Type P53
}

\author{
Robert F. H. Walter, ${ }^{1,2}$ Robert Werner, ${ }^{3}$ Michael Wessolly $\left(\mathbb{D},{ }^{1}\right.$ \\ Elena Mairinger, ${ }^{1,2}$ Sabrina Borchert $\mathbb{D}^{1},{ }^{1}$ Jan Schmeller $\mathbb{D}^{1},{ }^{1}$ Jens Kollmeier, ${ }^{4}$ \\ Thomas Mairinger, ${ }^{3}$ Thomas Hager, ${ }^{1}$ Agnes Bankfalvi, ${ }^{1}$ Daniel C. Christoph, ${ }^{5,6}$ \\ Wilfried E. E. Eberhardt, ${ }^{2,5}$ Till Plönes, ${ }^{7}$ Clemens Aigner, ${ }^{7}$ Kurt W. Schmid, ${ }^{1}$ \\ Jeremias Wohlschlaeger, ${ }^{1,8}$ and Fabian D. Mairinger (D) ${ }^{1}$ \\ ${ }^{1}$ Institute of Pathology, University Hospital Essen, University of Duisburg-Essen, Essen, Germany \\ ${ }^{2}$ Ruhrlandklinik, West German Lung Center, University Hospital Essen, University of Duisburg-Essen, Germany \\ ${ }^{3}$ Department of Pathology, Helios Klinikum Emil von Behring, Berlin, Germany \\ ${ }^{4}$ Department of Pneumology, Helios Klinikum Emil von Behring, Berlin, Germany \\ ${ }^{5}$ Department of Medical Oncology, West German Cancer Center, University Hospital Essen, \\ University of Duisburg-Essen, Essen, Germany \\ ${ }^{6}$ Department of Internistic Oncology, Kliniken Essen Mitte, Essen, Germany \\ ${ }^{7}$ Department of Thoracic Surgery and Thoracic Endoscopy, Ruhrlandklinik, \\ University Hospital Essen, University of Duisburg-Essen, Essen, Germany \\ ${ }^{8}$ Department of Pathology, Diakonissenkrankenhaus Flensburg, Flensburg, Germany
}

Correspondence should be addressed to Fabian D. Mairinger; fabian.mairinger@uk-essen.de

Received 14 February 2018; Revised 23 May 2018; Accepted 11 June 2018; Published 17 July 2018

Academic Editor: Akira Iyoda

Copyright (C) 2018 Robert F. H. Walter et al. This is an open access article distributed under the Creative Commons Attribution License, which permits unrestricted use, distribution, and reproduction in any medium, provided the original work is properly cited.

Previously, our group demonstrated that nuclear expression of E3 ubiquitin ligase (MDM2) in malignant pleural mesothelioma (MPM) is significantly associated with decreased overall survival. A possible explanation may be that overexpression of MDM2 leads to a proteasomal degradation of TP53 that eventually results in a loss of TP53-induced apoptosis and senescence. It is well known from other tumor entities that restoration of TP53 activity, e.g., by MDM2 inhibition, results in an instant TP53-induced stress and/or DNA damage response of cancer cells. Nutlin-3A (a cis-imidazoline analogue) has been described as a potent and selective MDM2 inhibitor preventing MDM2-TP53-interaction by specific binding to the hydrophobic TP53-binding pocket of MDM2. In the present study, the effects of MDM2 inhibition in MPM via Nutlin-3A and standard platinum based chemotherapeutic agents were comparatively tested in three MPM cell lines (NCI-H2052, MSTO-211H, and NCI-H2452) showing different expression profiles of TP53, MDM2, and its physiological inhibitor of MDM2-P14/ARF. Our in vitro experiments on MPM cell lines revealed that Nutlin-3A in combination with cisplatin resulted in up to 9.75 times higher induction of senescence $(\mathrm{p}=0.0050)$ and up to 5 times higher apoptosis rate $(\mathrm{p}=0.0067)$ compared to the commonly applied cisplatin and pemetrexed regimens. Thus Nutlin-3A, a potent inhibitor of MDM2, is associated with a significant induction of senescence and apoptosis in MPM cell lines, making Nutlin-3A a promising substance for a targeted therapy in the subgroup of MPM showing MDM2 overexpression.

\section{Introduction}

Malignant mesothelioma is a highly aggressive tumor arising from mesothelial lined surfaces, mostly from the pleural cavities (malignant pleural mesothelioma, MPM) [1, 2]. When untreated, the median survival of patients is nine months [35]. MPM patients are negatively affected by mostly insufficient current treatment modalities consisting of platinumcontaining regimes using cisplatin [6] or carboplatin [7-10] 
TABLE 1: Concentrations for each cytostatic substance and combination applied.

\begin{tabular}{lc}
$\begin{array}{l}\text { Testing Nutlin concentrations in comparison to Pemetrexed and } \\
\text { Cisplatin }\end{array}$ & $\begin{array}{c}\text { Testing Nutlin in combination with Cisplatin in comparison to } \\
\text { Pemetrexed and Cisplatin }\end{array}$ \\
\hline 10nM Cisplatin & 10nM Cisplatin \\
\hline 200nM Pemetrexed & 200nM Pemetrexed \\
\hline 10nm Cisplatin + 200nM Pemetrexed & 10nm Cisplatin + 200nM Pemetrexed \\
\hline 5nM Nutlin & 10nm Cisplatin + 5nM Nutlin \\
\hline 10nM Nutlin & 10nm Cisplatin + 10nM Nutlin \\
\hline 20nM Nutlin & 10nm Cisplatin + 20nM Nutlin \\
\hline
\end{tabular}

as first choice. Cisplatin treatment results in a response rate of merely $14 \%$ and a median survival of less than seven months [11]. Carboplatin resulted in similar response rates ranging from 6 to $16 \%[11,12]$. In clinical practice, the antifolate pemetrexed, as the only FDA-approved therapeutic for MPM, is used in combination with platin compounds [6-10].

Several studies have shown the efficacy of the evaluation of intratumoral expression of members of the folic acid metabolism for prediction of multitargeted antifolate therapy response in patients with different cancer entities but are discussed controversially [10, 13-28]. As platin-analoga are genotoxic compounds that induce DNA damage [29] leading to TP53 induced cell cycle arrest and apoptosis [30], it is basically conceivable that the DNA repair mechanism might be one of the keys associated with an impaired therapy response. As the identification of molecular properties shared by MPMs may help to overcome the poor treatment response observed, several studies addressed this question [11, 12, 27, 31-34]. However, the reasons for the rather poor efficacy of platinum compounds remain largely unknown.

Summing up, neither reliable predictive biomarkers nor individualized therapeutic concepts for MPM exist until now. Therefore, current guidelines emphasize the need of innovative and novel therapies [35].

Since mutations of the TP53 gene are extremely rare in MPM [36-38], other mechanisms such as deletion of the locus or epigenetic alterations may contribute to inactivation of TP53 [36-38]. Overexpression of MDM2 in some tumor types can lead to a loss of TP53 regulatory function in cancer cells by its increased proteasomal degradation [39-44]. P14/ARF, the physiological inhibitor of MDM2, is recognized as a tumor suppressor and contributes to this mechanism by induction of cell cycle arrest in both a TP53-dependent and TP53-independent manner. Moreover, miRNA regulation seems to play an important role [4552]. In previous studies, we have demonstrated a strong nuclear MDM2 overexpression in approximately $25 \%$ of MPM; this observation was restricted to epithelioid MPM or the epithelioid components of biphasic MPM [44, 53]. Patients with MDM2-positive MPM showed a significantly decreased overall survival (OS) and progression-free survival (PFS) compared to MDM2-negative MPM [44, 53]. This might be explained by a significantly decreased or completely abolished TP53 activity and/or stability mediated by an overexpression of MDM2 [39-43].

A restoration of TP53 activity, e.g., by MDM2 inhibition, might result in an instant TP53 induced stress and/or DNA damage response of cancer cells. Nutlin-3A (a cis-imidazoline analogue) is a potent and selective MDM2 inhibitor with an $\mathrm{IC}_{50}$ value of $90 \mathrm{nM}$ [54] and prevents MDM2-TP53interaction by binding to the hydrophobic TP53-binding pocket of MDM2 [55].

Thus, the aim of this study was to test the effect of MDM2 inhibition in MPM via Nutlin-3A in comparison to the contemporary common chemotherapeutic strategies using three cell lines showing different marker profiles concerning TP53-status, P14/ARF- and MDM2 expression level.

\section{Material and Methods}

2.1. Cell Line Experiments. Based on reviewing the literature, concentrations for the cytostatics were estimated (Nutlin-3A $[55,56]$, cisplatin [57], and pemetrexed [57], respectively).

Human MPM cell lines were obtained from the American Type Culture Collection in 2012-08 (Manassas, VA, USA). The cell lines were authenticated and tested for contaminations by using a commercial service (Multiplexion, Heidelberg, Germany) and were last retested directly after the experiments were finished.

NCI-H2052, NCI-H2452, and MSTO-211H were cultured in Roswell Park Memorial Institute (RPMI) medium (Invitrogen, CA, USA) containing 10\% fetal bovine serum (Invitrogen) at $37^{\circ} \mathrm{C}$ in a $5 \% \mathrm{CO}_{2}$-humidified atmosphere. Cells were grown until $85 \%$ to $95 \%$ confluency, then washed with phosphate-buffered saline (Invitrogen), and trypsinized with $1 \mathrm{ml}$ of $0.05 \%$ trypsin- $0.53 \mathrm{mM}$ ethylenediaminetetraacetic acid, phenol red (Invitrogen). Trypsinization was stopped by adding fresh medium to the reaction. Approximately $10 \mu \mathrm{l}$ was transferred to a hemocytometer (BRAND, Wertheim, Germany) for cell counting purposes. 1,000 cells per well $(100 \mu \mathrm{l})$ were seeded into microplates 96/U (Eppendorf, Hamburg, Germany) suitable for luminescence and fluorescence detection. The cells were allowed to attach overnight at $37^{\circ} \mathrm{C}$ and $5 \% \mathrm{CO}_{2}$. At the next day, the medium was removed and fresh medium containing either one of the cytostatics or without additive was applied to each well. Cisplatin $(10 \mu \mathrm{M}$; TEVA, Petah Tikva, Israel) pemetrexed $(200 \mu \mathrm{M}$; Lilly, IN, USA) and Nutlin-3A $(5,10$ or $20 \mu \mathrm{M}$; Sigma-Aldrich, MO, USA) was applied either alone or in combination. Nutlin-3A had to be solubilized in dimethyl sulfoxide (Sigma-Aldrich). Concentrations of the applied cytostatics are summarized in Table 1. Cell cultures containing cytostatics and blank medium were incubated for three days at $37^{\circ} \mathrm{C}$ and $5 \% \mathrm{CO}_{2}$. Within 72 hours, necrosis, 
TABLE 2: Molecular marker constellation of the investigated MPM cell lines. The immunoexpression or mRNA-expression of the investigated markers is shown for each investigated cell line.

\begin{tabular}{|c|c|c|c|}
\hline Cell line & MDM2 & P53 & P14/ARF \\
\hline NCI-H2052 & “+” & “+” & "+l-" \\
\hline MSTO-211H & “+/-” & “+” & “_” \\
\hline NCI-H2452 & “_” & “_” & “+” \\
\hline
\end{tabular}

-: minimal to no expression

+: expression measurable

+/-: little expression measurable

apoptosis, and cell viability were assessed by using the following luminescence assays: CytoTox-Glo ${ }^{\mathrm{TM}}$ Cytotoxicity Assay (Promega), Caspase-Glo ${ }^{\circledR}$ 3/7 Assay (Promega), and CellTiter-Glo ${ }^{\otimes}$ Luminescent Cell Viability Assay (Promega). The assays were performed as recommended by the supplier. Per cytostatic drug and luminescence assay at least four data points were measured. Luminescence was assessed using a SpectraMax L Luminescence Microplate Reader (Molecular Devices, CA, USA). Luminescence (relative luminescent units; RLU) was measured at $570 \mathrm{~nm}$ and integration time was adjusted to 1 second. Temperature of the SpectraMax L was kept between $21.5^{\circ} \mathrm{C}$ and $24.5^{\circ} \mathrm{C}$ during measurements. Additionally, from each cell line a FFPE block was prepared for immunohistochemical and qPCR analysis.

2.2. RNA Isolation and Real-Time qPCR. Expression levels of $A C T B$ (reference gene), MDM2 and P14/ARF, were investigated by TaqMan real-time qPCR in the three MPM cell lines. Therefore, RNA was isolated by cutting three to five sections of $4 \mu \mathrm{m}$ from the FFPE block using a microtome (Leica, SM 2000 R, Wetzlar, Germany). Total RNA was isolated using the miRNeasy FFPE kit (Qiagen, Hilden, Germany) and manufacturer's protocol, except for two modifications (proteinase $\mathrm{K}$ digestion overnight; elution in $25 \mu \mathrm{l}$ ). RNA concentrations were measured using UV/VIS spectrometry (NanoDrop ND-1000, PEQLAB Biotechnologie GmbH, Erlangen, Germany). RNA was stored at $-80^{\circ} \mathrm{C}$. For cDNA synthesis, the iScript Select cDNA Synthesis Kit and protocol (Bio-Rad Laboratories, Inc., CA, USA) was used with an input of $1 \mu \mathrm{g}$ total RNA per reaction.

For real-time qPCR, the TaqMan Gene Expression Assays on Demand (AoD) for ACTB (Hs03023943_g1), MDM2 (Hs01066942_ml), and P14/ARF (Hs99999189_ml) were used (Applied Biosystems ${ }^{\circledR} ;$ CA, USA). The reaction volumes were modified by using $50 \%$ of the recommended total reaction volumes with $50 \mathrm{ng}$ cDNA input. Each target was measured in triplicate. Ct-values of P14/ARF and MDM2 were normalized to the mean values of $A C T B$. Real-time qPCR and data analysis were performed on a Roche LightCycler 480 II (Roche, Basel, Switzerland) and corresponding software. All real-time qPCR experiments were performed in accordance with the MIQE-guidelines [58].

2.3. Immunohistochemistry. Immunohistochemistry was performed according to standard protocols using an automated stainer (Ventana Discovery XT, Munich, Germany). After validation on reference tissues (liposarcoma for MDM2, pulmonary adenocarcinoma for TP53), the immunohistochemical investigations were performed with antibodies directed against MDM2 (clone IF2, Calbiochem, Darmstadt, Germany, dilution: 1:80) and TP53 (clone BP53-12, Zytomed, Berlin, Germany; dilution: 1:5000). Pretreatment for antigen retrieval was performed by heating in deionized water at $\mathrm{pH}$ 6 for 30 minutes. Protein expression was assessed using a four-stage IHC scoring system based on the percentage of tumor cell nuclei with a positive immunoreaction (Score 0: no signal; Score 1 (weak expression): 1-25\%; Score 2 (moderate expression): $26-50 \%$; Score 3 (strong expression): $>50 \%$ ).

2.4. Statistical Analysis. Statistical and graphical analyses were performed with the $\mathrm{R}$ statistical programming environment (v3.4.2).

For analysis between single groups, either the Wilcoxon Mann-Whitney rank sum test (non-parametric) or twosided students t-test (parametric) was applied. For ordinal variables with more than two groups (luminescence signal differences between all treatment groups), either the KruskalWallis test (non-parametric) or ANOVA (parametric) was used to detect group differences.

The level of statistical significance was defined as $p<0.05$.

\section{Results}

The expression profiles of MDM2, TP53, and P14/ARF differ between the investigated cell lines and are summarized in Table 2. Scans of immunohistochemical staining's are shown in Figure 1; qPCR results are visualized in Figure 2. NCIH2052 showed pronounced MDM2-immunoexpression, but little P14/ARF and TP53-expression. Immunohistochemically, MSTO- $211 \mathrm{H}$ showed no expression of MDM2 and P14/ARF, but TP53-expression was present. NCI-H2452 showed neither MDM2- nor TP53-expression, but P14/ARF expression was detected. The investigated cell lines represent the molecular constellation that was reported in previous studies of patients with MPM $[59,60]$.

3.1. Response of MPM Cell Lines to Pemetrexed, Cisplatin, and Varying Nutlin-3A Concentrations. Cisplatin $(10 \mu \mathrm{M})$ and pemetrexed $(200 \mu \mathrm{M})$ as single agent as well as in combination were tested versus three Nutlin-3A concentrations $(5 \mu \mathrm{M}$, $10 \mu \mathrm{M}$, and $20 \mu \mathrm{M})$.

\subsubsection{Cell Viability}

NCI-H2052. Any Nutlin-3A concentration was superior in reducing cell viability compared to either cisplatin or pemetrexed or their combination, respectively $(\mathrm{p}=0.0039)$. In 


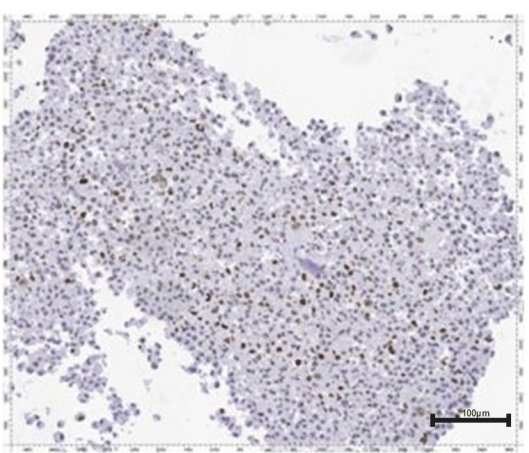

(a) NCI-H2052, anti-P53 staining

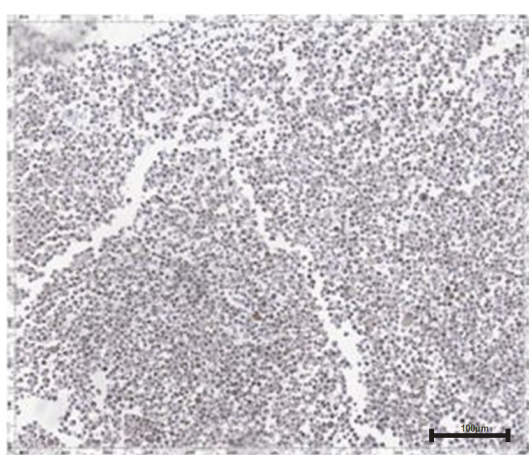

(d) NCI-H2452, anti-MDM2 staining

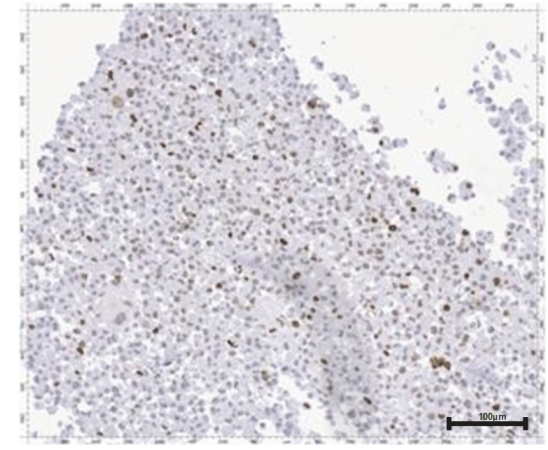

(b) NCI-H2052, anti-MDM2 staining

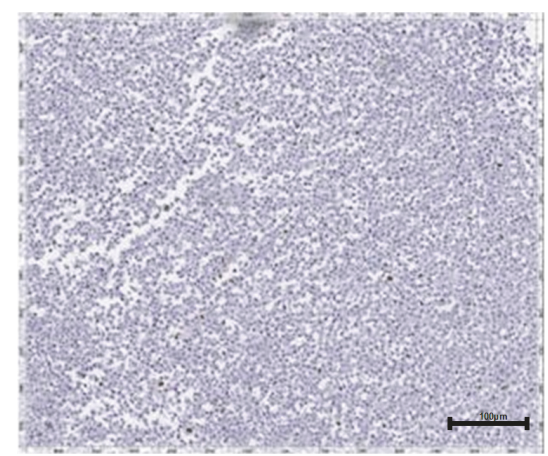

(e) MSTO-211H, anti-P53 staining

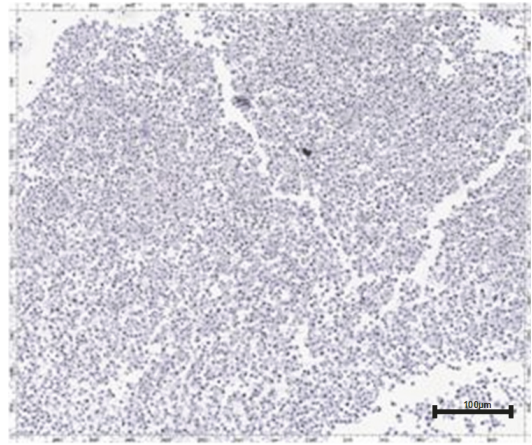

(c) NCI-H2452, anti-P53 staining

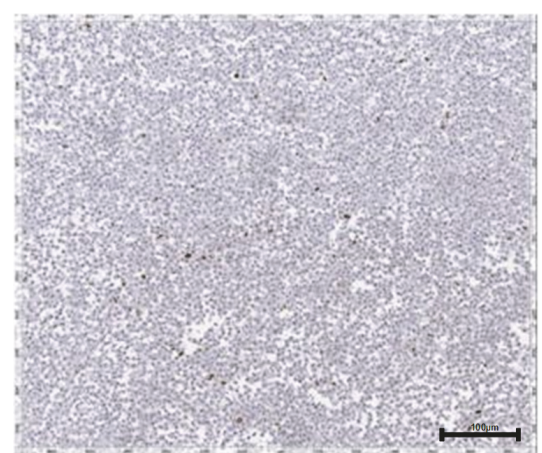

(f) MSTO-211H, anti-MDM2 staining

FIGURE 1: Immunohistochemical staining of the investigated MPM cell lines with antibodies directed against P53 and MDM2. NCIH2052 shows a strong staining (Score 2) regarding P53 (a) and MDM2 (b). NCI-H2452 showed neither immunoexpression for P53 ((c), Score 0) nor for MDM2 ((d), Score 0). MSTO-211H stained positive for P53 ((e), Score 1) and MDM2 ((f), Score 1). The scale bars indicate $100 \mu \mathrm{m}$ for pictures (a) and (b) and $500 \mu \mathrm{m}$ for pictures (c), (d), (e) and (f).

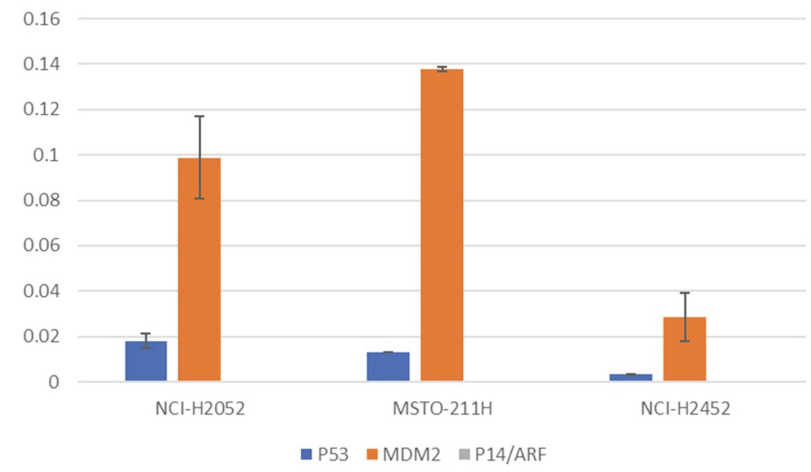

FIGURE 2: The bar chart shows the relative mRNA expression of MDM2, P53 and P14/ARF in the investigated MPM cell lines. On the $\mathrm{x}$-axis the investigated cell lines are shown and the respective mRNA expression of $P 53, M D M 2$, and $P 14 / A R F$. On the $\mathrm{y}$-axis the $2 \wedge \triangle \mathrm{Ct}$ values for the relative mRNA expression of the investigated target genes is shown after normalization against the reference gene $A C T B$ (actin, beta). NCI-H2052 and MSTO-211H show elevated expression of MDM2, whereas NCI-H2452 showed minimal MDM2 expression. TP53 mRNA expression was reduced in NCI-H2452 compared to both other cell lines. P14/ARF expression was below the detection limit in the investigated specimens.

contrast, treatment with pemetrexed alone showed significantly elevated cell viability. Treatment with cisplatin alone showed higher cell viability than cisplatin and pemetrexed in combination.

MSTO-211H. Pemetrexed combined with cisplatin was associated with the highest cell viability, followed by cisplatin alone and the lowest Nutlin-3A concentration ( $\mathrm{p}=0.0952)$.
Pemetrexed combined with cisplatin reduced cell viability significantly, but Nutlin-3A $(10 \mu \mathrm{M})$ exhibited a slightly stronger reduction. The highest Nutlin-3A concentration reduced cell viability to a minimum.

NCI-H2452. The highest Nutlin-3A concentration $(20 \mu \mathrm{M})$ reduced cell viability to a minimum $(\mathrm{p}=0.0017) .10 \mu \mathrm{M}$ Nutlin$3 \mathrm{~A}$ was the second strongest cell viability inhibitor followed 


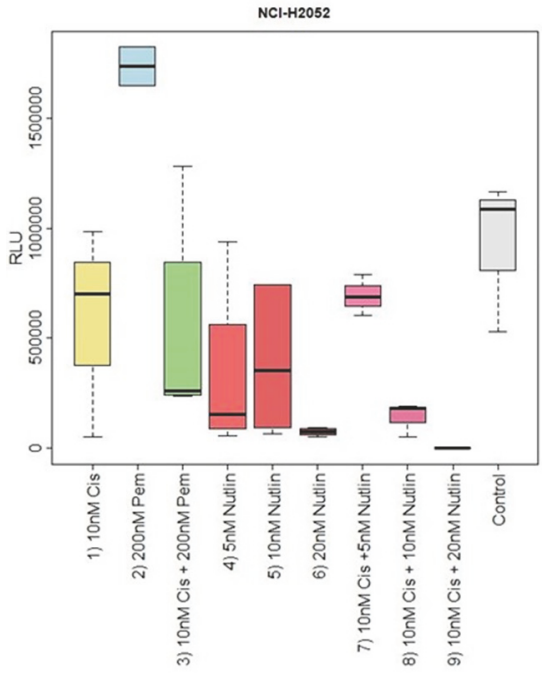

(a)

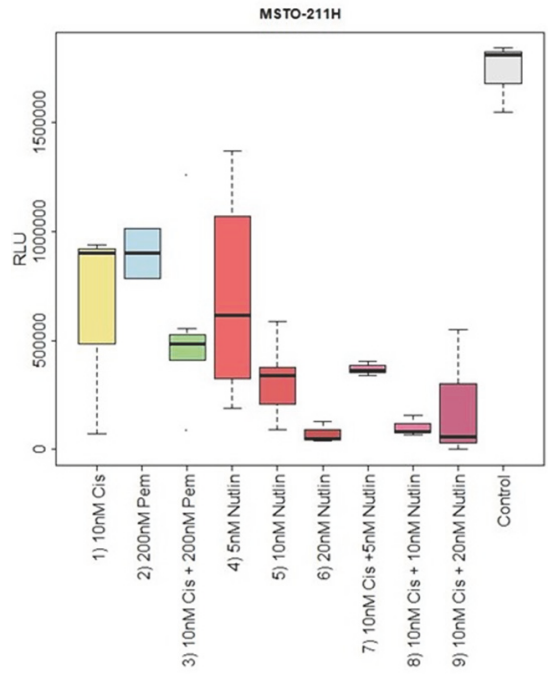

(b)

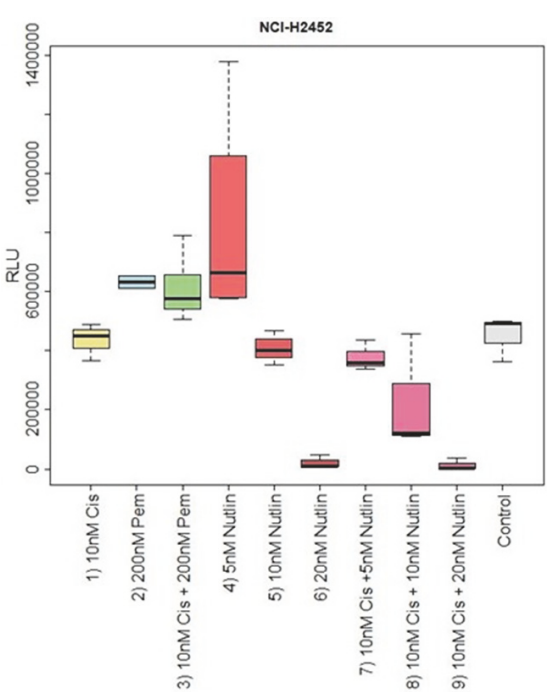

(c)

FIGURE 3: Induction of senescence in MPM cell lines by pemetrexed, cisplatin, and varying Nutlin-3A concentrations as well as varying Nutlin-3A concentrations combined with cisplatin. Figure 3 shows boxplots for cell viability/senescence for the three investigated MPM cell lines. On the y-axis RLU (relative luminescence units) are shown. High RLU indicate high cell viability, whereas low RLU indicate senescence. On the $\mathrm{x}$-axis, the concentrations of the cytostatics applied and the control are shown. In all three MPM cell lines, $20 \mu \mathrm{M}$ Nutlin-3A showed the strongest inhibition of cell viability compared to the other single agent cytostatics and concentrations applied. This is true against other Nutlin3 A concentrations (NCI-H2052: $\mathrm{p}=0.021$, MSTO-211H: $\mathrm{p}=0.007$, NCI-H2452: $\mathrm{p}<0.001$ ), cisplatin (NCI-H2052: $\mathrm{p}=0.021, \mathrm{MSTO}-211 \mathrm{H}: \mathrm{p}=0.018$, NCI-H2452: $\mathrm{p}=0.004)$, pemetrexed (NCI-H2052: $\mathrm{p}=0.032$, MSTO-211H: $\mathrm{p}=0.008$, NCI-H2452: $\mathrm{p}=0.006$ ), and a combination of both (NCIH2052: $\mathrm{p}=0.003$, MSTO-211H: $\mathrm{p}=0.002$, NCI-H2452: $\mathrm{p}<0.001)$. Additionally, higher concentrations of Nutlin-3A $(10 \mu \mathrm{M}, 20 \mu \mathrm{M})$ combined with cisplatin regimen showed the strongest inhibition of cell viability compared to nowadays approved cytostatics, either as single agents (cisplatin: NCI-H2052: $\mathrm{p}=0.021$, MSTO-211H: $\mathrm{p}=0.022$, NCI-H2452: $\mathrm{p}=0.006$; pemetrexed: NCI-H2052: $\mathrm{p}=0.014$, MSTO-211H: $\mathrm{p}=0.029, \mathrm{NCI}$ H2452: $\mathrm{p}<0.001$ ) or in combination (NCI-H2052: $\mathrm{p}=0.003$, MSTO-211H: $\mathrm{p}=0.014$, NCI-H2452: $\mathrm{p}<0.001$ ).

by cisplatin alone, pemetrexed alone, and cisplatin in combination with pemetrexed. The lowest Nutlin-3A concentration showed the weakest impact on cell viability reduction.

Box plots for cell viability highlight decreasing cell viability with increasing Nutlin-3A concentration in the tested cell lines. The results for all cell lines regarding senescence/cell viability are summarized in Figures 3(a)-3(c).

\subsubsection{Apoptosis}

NCI-H2052. In the NCI-H2052 cell line, the highest apoptosis rate was found for $20 \mu \mathrm{M}$ Nutlin-3A, whereas the other treatment approaches showed similar apoptosis induction $(\mathrm{p}=0.14)$.

MSTO-211H. In MSTO-211H, highest apoptosis rates were found for pemetrexed followed by pemetrexed in combination with cisplatin and different Nutlin-3A concentrations $(\mathrm{p}=0.0219)$. Almost no apoptosis was observed for cisplatin alone and Nutlin-3A.

NCI-H2452. NCI-H2452 revealed the highest apoptosis rate in response to Nutlin-3A in the highest concentration $(20 \mu \mathrm{M})$ followed by cisplatin $(\mathrm{p}=0.0359)$. Significantly lower apoptosis rates were found for the remaining cytostatics.
The results for apoptosis are summarized in Figures 4(a) $-4(\mathrm{c})$.

3.1.3. Necrosis. Necrosis of cells was not influenced by any of the chemotherapeutics compared to the control (data not shown).

3.2. Response of MPM Cell Lines to Varying Nutlin-3A Concentrations Combined with Cisplatin. In further experiments, the induction of apoptosis was tested by using either a Nutlin$3 \mathrm{~A}$ regimen or a combination of Nutlin-3A and cisplatin. Three combinations of Nutlin-3A $(5 \mu \mathrm{M}, 10 \mu \mathrm{M}$, and $20 \mu \mathrm{M})$ plus cisplatin $(10 \mu \mathrm{M})$ were compared with cisplatin $(10 \mu \mathrm{M})$ alone, pemetrexed $(200 \mu \mathrm{M})$ alone, Nutlin-3A alone $(10 \mu \mathrm{M})$, and a combination of cisplatin and pemetrexed.

\subsubsection{Cell Viability}

NCI-H2052. Nutlin-3A alone and its combination with cisplatin showed significantly increased induction of senescence compared to the other regimen $(\mathrm{p}=0.0051)$. Only $5 \mu \mathrm{M}$ Nutlin-3A in combination with cisplatin showed lower potency to induce senescence rates as $5 \mu \mathrm{M}$ Nutlin-3A without cisplatin. The higher Nutlin-3A concentrations (10 and 


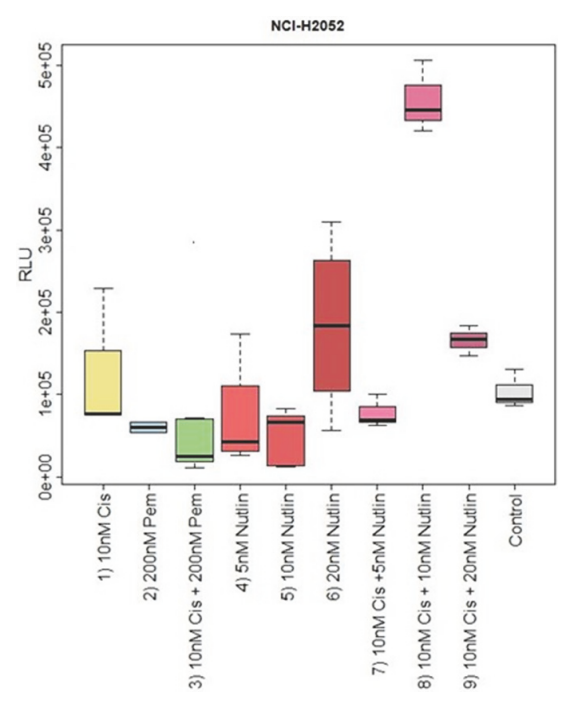

(a)

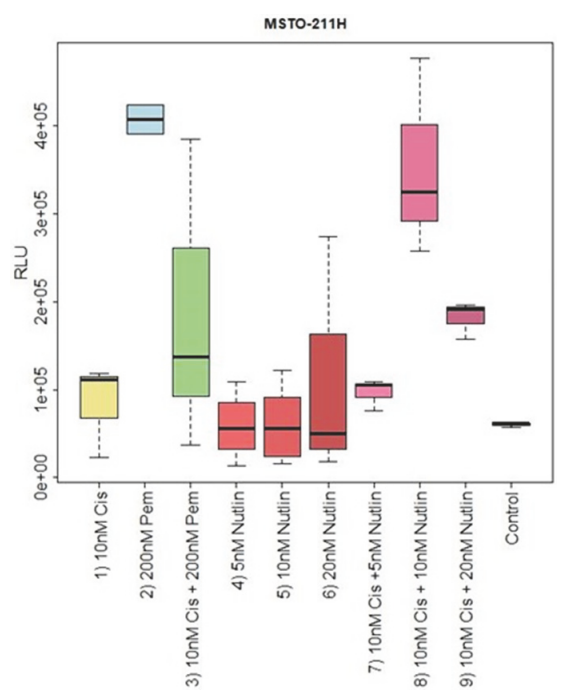

(b)

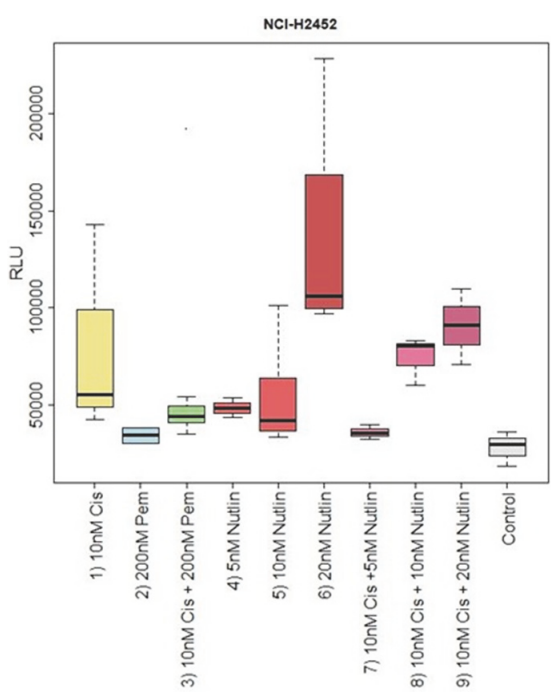

(c)

FIGURE 4: Figure 4 shows boxplots for apoptosis for the investigated MPM cell lines. On the y-axis RLU (relative luminescence units) are shown. RLU and increasing apoptosis rates show a direct correlation. On the X-axis, the concentrations of the cytostatics applied and the control are shown. For cell line NCI-H2052 and NCI-H2452 (shown in Figures 4(a) and 4(c), respectively), 20 4 M Nutlin-3A showed the strongest induction of apoptosis when comparing single agents (cisplatin: NCI-H2052: $\mathrm{p}=0.084$, NCI-H2452: $\mathrm{p}=0.028$; pemetrexed: NCIH2052: $\mathrm{p}=0.011$, NCI-H2452: $\mathrm{p}=0.049$ ) but also against cisplatin combined with pemetrexed (NCI-H2052: $\mathrm{p}=0.015, \mathrm{NCI}-\mathrm{H} 2452$ : $\mathrm{p}=0.008$ ). MSTO-211H (shown in Figure 4(b)), the highest apoptosis rate, was found for pemetrexed followed by $5 \mu \mathrm{M}, 20 \mu \mathrm{M}$ Nutlin-3A, and the combination of pemetrexed and cisplatin (all p<0.001). When analyzing Nutlin-3A in combination with cisplatin, for cell line NCI-H2052, (a) the highest apoptosis rate was found for $10 \mu \mathrm{M}$ Nutlin-3A combined with cisplatin (all p<0.001). MSTO-211H (b) apoptotic rates of $10 \mu \mathrm{M}$ Nutlin-3A combined with cisplatin comparable to the treatment with pemetrexed alone $(\mathrm{p}=0.493)$ significantly enhanced against all other approaches $(\mathrm{p}=0.016)$. In NCI-H2452 (c), treatment with cisplatin combined with $20 \mu \mathrm{M}$ and $10 \mu \mathrm{M}$ Nutlin $3 \mathrm{~A}$ showed the strongest induction of apoptosis beside $20 \mu \mathrm{M}$ Nutlin-3A alone $(\mathrm{p}=0.004)$ but shows no statistically significant differences compared with $20 \mu \mathrm{M}$ Nutlin-3A single agent treatment $(\mathrm{p}=0.199)$.

$20 \mu \mathrm{M})$ with cisplatin reduced cell viability to a minimum. The highest cell viability was found for pemetrexed followed by the combination of pemetrexed and cisplatin.

MSTO-211H. Any combination of Nutlin-3A with cisplatin induced significantly increased cellular senescence compared to cisplatin, pemetrexed, or a combination of both $(p=0.0059)$. However, the combination of cisplatin and pemetrexed showed similar efficacy compared to the lowest Nutlin-3A/cisplatin regimen and Nutlin-3A alone. Higher concentrations of Nutlin-3A combined with cisplatin reduced cell viability to a minimum.

NCI-H2452. Nutlin-3A in combination with cisplatin or alone was superior compared to the other cytostatics, except at the lowest concentration of $5 \mu \mathrm{M}(\mathrm{p}=0.0089)$. Interestingly, cisplatin showed comparable efficacy as $10 \mu \mathrm{M}$ Nutlin-3A alone and cisplatin in combination with $5 \mu \mathrm{M}$ Nutlin-3A. The highest cell viability was observed with pemetrexed, cisplatin in combination with pemetrexed, and $5 \mu \mathrm{M}$ Nutlin-3A. The highest Nutlin-3A concentration $(20 \mu \mathrm{M})$ with cisplatin showed the highest senescence rate.

Box plots for cell viability highlight that cell viability decreased with increasing concentration of the cisplatin/ Nutlin-3A regimen in the tested cell lines. The results for senescence/cell viability are summarized in Figures 3(a)-3(c).

\subsubsection{Apoptosis}

NCI-H2052. In the NCI-H2052 cell line, higher Nutlin-3A concentrations combined with cisplatin applied induced significantly increased apoptosis compared to pemetrexed alone or combined with cisplatin ( $\mathrm{p}=0.0069)$. The highest apoptosis rates were found for $10 \mu \mathrm{M}$ Nutlin-3A in combination with cisplatin.

MSTO-211H. Cell line MSTO-211H exhibited the highest apoptosis when treated with pemetrexed alone $(\mathrm{p}=0.0035)$. The second highest apoptosis rate was found for $10 \mu \mathrm{M}$ Nutlin-3A combined with cisplatin. Pemetrexed in combination with cisplatin resulted in the third highest apoptosis rate. Cisplatin in combination with $20 \mu \mathrm{M}$ Nutlin-3A was more potent than cisplatin alone, Nutlin-3A alone, and the lowest concentration of Nutlin-3A $(5 \mu \mathrm{M})$ in combination with cisplatin.

NCI-H2452. The highest apoptosis rates were found for the $20 \mu \mathrm{M}$ Nutlin-3A single agent as well as $10 \mu \mathrm{M}$ and $20 \mu \mathrm{M}$ Nutlin-3A concentrations combined with cisplatin, followed by cisplatin $(\mathrm{p}=0.1)$.

The results regarding apoptosis are summarized in Figures $4(\mathrm{a})-4(\mathrm{c})$. 
TABLE 3

(a) Response of MPM cell lines to pemetrexed, cisplatin, and varying Nutlin-3A concentrations

\begin{tabular}{|c|c|c|c|c|c|c|}
\hline & \multicolumn{6}{|c|}{ Cell viability } \\
\hline & 10um Cis & 200um Pem & 10um Cis +200 um Pem & 5um Nut & 10uM Nut & 20uM Nut \\
\hline $\mathrm{H} 2052$ & + & - & + & ++ & +++ & +++ \\
\hline MSTO-211H & 0 & + & 0 & 0 & ++ & +++ \\
\hline \multirow[t]{3}{*}{ H2452 } & 0 & 0 & - & - & + & +++ \\
\hline & \multicolumn{6}{|c|}{ Apoptosis } \\
\hline & 10um Cis & 200um Pem & 10um Cis+200um Pem & 5um Nut & 10uM Nut & 20uM Nut \\
\hline $\mathrm{H} 2052$ & 0 & + & 0 & 0 & 0 & ++ \\
\hline MSTO-211H & 0 & ++ & + & + & 0 & ++ \\
\hline $\mathrm{H} 2452$ & 0 & 0 & 0 & 0 & ++ & +++ \\
\hline
\end{tabular}

(b) Response of MPM cell lines to varying Nutlin-3A concentrations combined with cisplatin

Cell viability

\begin{tabular}{|c|c|c|c|c|c|c|c|}
\hline \multicolumn{8}{|c|}{ Cell viability } \\
\hline & 10uM Cis & 200um Pem & 10um Cis+200um Pem & 5um Nut+10um Cis & 10uM Nut+10um Cis & 20uM Nut+10um Cis & 10uM Nut \\
\hline H2052 & 0 & - & 0 & + & ++ & +++ & + \\
\hline MSTO-211H & + & + & ++ & ++ & +++ & +++ & ++ \\
\hline $\mathrm{H} 2452$ & + & - & - & + & ++ & +++ & + \\
\hline \multicolumn{8}{|c|}{ Apoptosis } \\
\hline & $10 \mathrm{uM} \mathrm{Cis}$ & 200um Pem & 10um Cis +200 um Pem & 5um Nut+10um Cis & 10uM Nut+10um Cis & 20uM Nut+10um Cis & 10uM Nut \\
\hline H2052 & 0 & 0 & 0 & 0 & +++ & ++ & 0 \\
\hline MSTO-211H & + & +++ & +++ & + & +++ & ++ & + \\
\hline $\mathrm{H} 2452$ & + & 0 & + & 0 & ++ & +++ & + \\
\hline
\end{tabular}

3.2.3. Necrosis. Necrosis of cells was not influenced by any of the chemotherapeutics compared to the non-treated control (data not shown).

All results of the cell line inhibition experiments are summarized in Table 3.

\section{Discussion}

In previous studies we identified MDM2 as a prognostic biomarker in patients with MPM and that expression is regulated through specific miRNA [44, 52, 59]. Nutlin-3A inhibits MDM2-TP53 interaction and thereby induces cell cycle arrest, senescence, and apoptosis depending on the cell type $[61,62]$. Additionally, it is a nongenotoxic drug that exhibits little toxicity in animal models and is associated with a lower risk of resistance than conventional drugs [61-63].

Against this background we hypothesized that MDM2 overexpression, maybe in combination with partial or complete loss of P14/ARF, can be targeted by a Nutlin-3A based therapy regimen to restore TP53 activity in a subgroup of MPM.

In this in vitro approach, the effects of the nowadays state-of-the-art chemotherapeutics cisplatin and pemetrexed, alone and in combination, compared to Nutlin-3A were investigated in three cell lines covering the pattern found in patients $[44,59]$. Nutlin-3A induced senescence efficiently in all three MPM cell lines and was superior compared to cisplatin and/or pemetrexed, whereas apoptosis could only be induced at high concentrations. It is known from the literature, that the effects of Nutlin-3A are cell type specific $[61,62,64]$, rather inducing cell cycle arrest and senescence than apoptosis [64]. Accordingly, we investigated cisplatin and Nutlin-3A in combination to increase cellular stress by inducing platin-based DNA damage. The combination of Nutlin-3A with cisplatin results in increased apoptosis and senescence rates compared to Nutlin-3A alone, as a major function of TP53 is DNA damage and stress response [46].

The same mechanism seems to be true when combining Nutlin-3A and radiotherapy to provide additional cellular damage and shift the cellular TP53-response towards apoptosis, already shown in TP53 wild-type esophageal squamous cell carcinoma in vitro and in vivo [65]. Interestingly, Shimazu et al. [66] found an additional growth inhibitory effect in MPM when combining Nutlin-3A with metformin, an mTOR inhibitor, suggesting a possible cross-talk between the mTOR- and TP53-pathway. Of note, the authors confirmed our findings of the cell lines NCI-H2052 and MSTO-211H as best responders to Nutlin-3A therapy, postulating an IC50 value of $0.37 \mu \mathrm{M}(\mathrm{MSTO}-211 \mathrm{H})$ and $0.50 \mu \mathrm{M}$ (NCI-H2052), respectively [66].

As mentioned before, overexpression of MDM2 can lead to a loss of P53 regulatory function via increased proteasomal degradation [39-44]. Besides its physiological inhibitor P14/ARF, analysis of the signalling relationship between these genes indicates an additional role of RB1 in this signalling network [45-51]. It has been shown, that, besides 
inhibition of the MDM2-TP53 interaction, Nutlin-3A also influences MDM2-RB1 interactions, making this a possible explanation for Nutlin-3A based TP53 independent effects [67].

Interestingly, even the low MDM2 expressing cell line MSTO-211H as well as the MDM2 and TP53 negative cell line NCI-H2452 shows reduced but clearly detectable, induction of apoptosis via Nutlin-3A combined with cisplatin. Also, immunohistochemically negative cells have, as reported previously [59], detectable gene expression pattern of MDM2, resulting in MDM2 protein concentrations below the detection limit of IHC. We hypothesize, as MDM2 driven regulation of TP53 is an essential mediator of apoptosis and cell state in a physiological situation, also inhibition of the TP53-MDM2 interaction at this low MDM2 levels will have a beneficial effect on cytotoxicity of platinum compounds, explaining the occurring side effects of Nutlin-3A therapy [68]. For NCI-H2452, a cell line with absent expression of TP53, the observed effect must be TP53 independently and is most likely based on RB1 inhibitory effects.

Currently, Nutlin-3A is administered per os as substance R05045337 in a multicentre phase I clinical trial for therapy of hematologic neoplasia [69]. Additionally, RG7112, a derivative of Nutlin-3A has entered phase I clinical trials in patients with liposarcomas that are TP53 wild-type tumors with amplified MDM2 [70]. In this clinical trial, RG7112 was administered per os in 20 patients in a neoadjuvant setting [68]. One patient showed partial remission and 14 showed stable disease, but all patients suffered from side effects as neutropenia [68]. A possible explanation might be the high doses of medication of $1440 \mathrm{mg} \mathrm{m}^{-2}$ day $^{-1}$ per os [68]. In previous in vivo studies, oral administration of Nutlin-3A showed several limitations as high input amounts of Nutlin$3 \mathrm{~A}(200-400 \mathrm{mg} / \mathrm{Kg})$ and difficulties in administering these high dosages [69]. It is noteworthy that efficient delivery systems were developed using polymers as poly(lactide-coglycolide) (PLGA) and monoclonal antibodies [69].

\section{Conclusion}

In this in vitro study, our hypothesis that MDM2-overexpressing MPM can be targeted by a Nutlin-3A based chemotherapy was proven. Particularly, for an optimal biomarker setting of MDM2-overexpression and low/absent P14/ARF expression, superior apoptosis and senescence rates were seen compared to the conventional chemotherapeutics. Even for a less optimal biomarker setting with minimal MDM2 expression, a favorable induction of apoptosis and senescence was obvious for Nutlin-3A in combination with cisplatin compared to the conventional drug regimen. Therefore, Nutlin-3A based therapy approach could be of great value for a subgroup of patients with MPM.

\section{Data Availability}

The data used to support the findings of this study are available from the corresponding author upon request.

\section{Disclosure}

The results of the present study have been presented at the German Cancer Consortium (DKTK), 1st Essen Translational Oncology Symposium (ETOS) (Essen, 2018), and the 33rd German Cancer Congress (Berlin 2018).

\section{Conflicts of Interest}

The authors declare no conflicts of interest.

\section{Acknowledgments}

The study was financed by the Institute of Pathology, University Hospital Essen, and Ruhrlandklinik Essen.

\section{References}

[1] R. K. Goudar, "Review of pemetrexed in combination with cisplatin for the treatment of malignant pleural mesothelioma," Therapeutics and Clinical Risk Management, vol. 4, no. 1, pp. 205-211, 2008.

[2] Z. Liu and J. Klominek, "Regulation of matrix metalloprotease activity in malignant mesothelioma cell lines by growth factors," Thorax, vol. 58, no. 3, pp. 198-203, 2003.

[3] K. H. Antman, "Clinical presentation and natural history of benign and malignant mesothelioma," Seminars in Oncology, vol. 8, no. 3, pp. 313-320, 1981.

[4] M. Hazarika, R. M. White Jr., B. P. Booth, Y. C. Wang, D. Y. Ham, C. Y. Liang et al., "Pemetrexed in malignant pleural mesothelioma," Clinical cancer research: an official journal of the American Association for Cancer Research, vol. 11, no. 3, pp. 982992, 2005.

[5] H. Weill, J. M. Hughes, and A. M. Churg, "Changing trends in US mesothelioma incidence," Occupational and Environmental Medicine, vol. 61, no. 5, pp. 438-441, 2004.

[6] J. E. Herndon II, M. R. Green, A. P. Chahinian, J. M. Corson, Y. Suzuki, and N. J. Vogelzang, "Factors predictive of survival among 337 patients with mesothelioma treated between 1984 and 1994 by the Cancer and Leukemia Group B," CHEST, vol. 113, no. 3, pp. 723-731, 1998.

[7] G. L. Ceresoli, P. A. Zucali, A. G. Favaretto et al., "Phase II study of pemetrexed plus carboplatin in malignant pleural mesothelioma," Journal of Clinical Oncology, vol. 24, no. 9, pp. 1443-1448, 2006.

[8] G. L. Ceresoli, P. A. Zucali, M. Mencoboni et al., "Phase II study of pemetrexed and carboplatin plus bevacizumab as first-line therapy in malignant pleural mesothelioma," British Journal of Cancer, vol. 109, no. 3, pp. 552-558, 2013.

[9] G. L. Ceresoli, B. Castagneto, P. A. Zucali et al., "Pemetrexed plus carboplatin in elderly patients with malignant pleural mesothelioma: Combined analysis of two phase II trials," British Journal of Cancer, vol. 99, no. 1, pp. 51-56, 2008.

[10] P. A. Zucali, E. Giovannetti, A. Destro et al., "Thymidylate synthase and excision repair cross-complementing group-1 as predictors of responsiveness in mesothelioma patients treated with pemetrexed/carboplatin," Clinical Cancer Research, vol. 17, no. 8, pp. 2581-2590, 2011.

[11] S. Tomek and C. Manegold, "Chemotherapy for malignant pleural mesothelioma: Past results and recent developments," Lung Cancer, vol. 45, pp. S103-S119, 2004. 
[12] S. Tomek, S. Emri, K. Krejcy, and C. Manegold, "Chemotherapy for malignant pleural mesothelioma: Past results and recent developments," British Journal of Cancer, vol. 88, no. 2, pp. 167174, 2003.

[13] F. Mairinger, C. Vollbrecht, I. Halbwedl et al., "Reduced folate carrier and folylpolyglutamate synthetase, but not thymidylate synthase predict survival in pemetrexed-treated patients suffering from malignant pleural mesothelioma," Journal of Thoracic Oncology, vol. 8, no. 5, pp. 644-653, 2013.

[14] L. Righi, M. G. Papotti, P. Ceppi et al., "Thymidylate Synthase but not excision repair cross-complementation group 1 tumor expression predicts outcome in patients with malignant pleural mesothelioma treated with pemetrexed-based chemotherapy," Journal of Clinical Oncology, vol. 28, no. 9, pp. 1534-1539, 2010.

[15] D. E. S. Lustgarten, C. Deshpande, C. Aggarwal et al., "Thymidylate synthase and folyl-polyglutamate synthase are not clinically useful markers of response to pemetrexed in patients with malignant pleural mesothelioma," Journal of Thoracic Oncology, vol. 8, no. 4, pp. 469-477, 2013.

[16] D. C. Christoph, B. R. Asuncion, C. Mascaux et al., "Folylpolyglutamate synthetase expression is associated with tumor response and outcome from pemetrexed-based chemotherapy in malignant pleural mesothelioma," Journal of Thoracic Oncology, vol. 7, no. 9, pp. 1440-1448, 2012.

[17] A. A. Adjei, "Pemetrexed (ALIMTA), a novel multitargeted antineoplastic agent," Clinical Cancer Research, vol. 10, no. 12, 2004.

[18] K. Kawakami, A. Ooyama, A. Ruszkiewicz et al., "Low expression of $\gamma$-glutamyl hydrolase mRNA in primary colorectal cancer with the CpG island methylator phenotype," British Journal of Cancer, vol. 98, no. 9, pp. 1555-1561, 2008.

[19] Y. Shintani, M. Ohta, H. Hirabayashi et al., "Thymidylate synthase and dihydropyrimidine dehydrogenase mRNA levels in tumor tissues and the efficacy of 5-fluorouracil in patients with non-small-cell lung cancer," Lung Cancer, vol. 45, no. 2, pp. 189-196, 2004.

[20] P. Ceppi, M. Volante, S. Saviozzi et al., "Squamous cell carcinoma of the lung compared with other histotypes shows higher messenger RNA and protein levels for thymidylate synthase," Cancer, vol. 107, no. 7, pp. 1589-1596, 2006.

[21] M. Takamura, Y. Nio, K. Yamasawa, M. Dong, K. Yamaguchi, and M. Itakura, "Implication of thymidylate synthase in the outcome of patients with invasive ductal carcinoma of the pancreas and efficacy of adjuvant chemotherapy using 5-fluorouracil or its derivatives," Anti-Cancer Drugs, vol. 13, no. 1, pp. 75-85, 2002.

[22] H. Shiga, E. I. Heath, A. A. Rasmussen et al., "Prognostic value of p53, glutathione S-transferase $\pi$, and thymidylate synthase for neoadjuvant cisplatin-based chemotherapy in head and neck cancer," Clinical Cancer Research : An Official Journal of The American Association for Cancer Research, vol. 5, no. 12, pp. 4097-4104, 1999.

[23] Y. Shirota, J. Stoehlmacher, J. Brabender et al., "ERCC1 and thymidylate synthase mRNA levels predict survival for colorectal cancer patients receiving combination oxaliplatin and fluorouracil chemotherapy," Journal of Clinical Oncology, vol. 19, no. 23, pp. 4298-4304, 2001.

[24] R. Nishimura, K. Nagao, H. Miyayama et al., "Thymidylate synthase levels as a therapeutic and prognostic predictor in breast cancer," Anticancer Reseach, vol. 19, no. 6C, pp. 5621-5626, 1999.

[25] C. J. Allegra, A. L. Parr, L. E. Wold et al., "Investigation of the prognostic and predictive value of thymidylate synthase, p53, and Ki-67 in patients with locally advanced colon cancer," Journal of Clinical Oncology, vol. 20, no. 7, pp. 1735-1743, 2002.

[26] C. J. Allegra, S. Paik, L. H. Colangelo et al., "Prognostic value of thymidylate synthase, Ki-67, and p53 in patients with Dukes' $\mathrm{B}$ and C colon cancer: a National Cancer Institute-National Surgical Adjuvant Breast and Bowel Project collaborative study," Journal of Clinical Oncology, vol. 21, no. 2, pp. 241-250, 2003.

[27] S. Ting, F. D. Mairinger, T. Hager et al., "ERCC1, MLH1, MSH2, MSH6, and $\beta$ iII-tubulin: Resistance proteins associated with response and outcome to platinum-based chemotherapy in malignant pleural mesothelioma," Clinical Lung Cancer, vol. 14, no. 5, pp. 558-567, 2013.

[28] F. D. Mairinger, C. Vollbrecht, E. Flom et al., "Folic acid phenotype (FAP) is a superior biomarker predicting response to pemetrexed-based chemotherapy in malignant pleural mesothelioma," Oncotarget, vol. 8, no. 23, pp. 37502-37510, 2017.

[29] Y. Jung and S. J. Lippard, "Direct cellular responses to platinuminduced DNA damage," Chemical Reviews, vol. 107, no. 5, pp. 1387-1407, 2007.

[30] M. Koch, M. L. Krieger, D. Stölting et al., "Overcoming chemotherapy resistance of ovarian cancer cells by liposomal cisplatin: Molecular mechanisms unveiled by gene expression profiling," Biochemical Pharmacology, vol. 85, no. 8, pp. 10771090, 2013.

[31] N. S. Kamal, J.-C. Soria, J. Mendiboure et al., "MutS homologue 2 and the long-term benefit of adjuvant chemotherapy in lung cancer," Clinical Cancer Research, vol. 16, no. 4, pp. 1206-1215, 2010.

[32] R. J. Kelly, E. Sharon, and R. Hassan, "Chemotherapy and targeted therapies for unresectable malignant mesothelioma," Lung Cancer, vol. 73, no. 3, pp. 256-263, 2011.

[33] R. F. H. Walter, C. Vollbrecht, R. Werner et al., "Screening of pleural mesotheliomas for DNA-damage repair players by digital gene expression analysis can enhance clinical management of patients receiving platin-based chemotherapy," Journal of Cancer, vol. 7, no. 13, pp. 1915-1925, 2016.

[34] F. D. Mairinger, R. Werner, E. Flom et al., "miRNA regulation is important for DNA damage repair and recognition in malignant pleural mesothelioma," Virchows Archiv, vol. 470, no. 6, pp. 627-637, 2017.

[35] P. Astoul, E. Roca, F. Galateau-Salle, and A. Scherpereel, "Malignant pleural mesothelioma: from the bench to the bedside," Respiration, vol. 83, no. 6, pp. 481-493, 2012.

[36] T. Papp, H. Schipper, H. Pemsel et al., "Mutational analysis of $\mathrm{N}$-ras, p53, p16INK4a, p14ARF and CDK4 genes in primary human malignant mesotheliomas," International Journal of Oncology, vol. 18, no. 2, pp. 425-433, 2001.

[37] T. Papp, H. Schipper, H. Pemsel et al., "Mutational analysis of the PTEN/MMAC1 tumour suppressor gene in primary human malignant mesotheliomas," Oncology Reports, vol. 8, no. 6, pp. 1375-1379, 2001.

[38] S. Toyooka, T. Kishimoto, and H. Date, "Advances in the molecular biology of malignant mesothelioma," Acta Medica Okayama, vol. 62, no. 1, pp. 1-7, 2008.

[39] S. N. Jones, A. E. Roe, L. A. Donehower, and A. Bradley, "Rescue of embryonic lethality in Mdm2-deficient mice by absence of p53," Nature, vol. 378, no. 6553, pp. 206-208, 1995.

[40] J.-C. Marine, S. Francoz, M. Maetens, G. Wahl, F. Toledo, and G. Lozano, "Keeping p53 in check: Essential and synergistic functions of Mdm2 and Mdm4," Cell Death \& Differentiation, vol. 13, no. 6, pp. 927-934, 2006. 
[41] R. M. D. O. Luna, D. S. Wagner, and G. Lozano, "Rescue of early embryonic lethality in mdm2-deficient mice by deletion of $\mathrm{p} 53$," Nature, vol. 378, no. 6553, pp. 203-206, 1995.

[42] J. Parant, A. Chavez-Reyes, N. A. Little et al., "Rescue of embryonic lethality in Mdm4-null mice by loss of Trp53 suggests a nonoverlapping pathway with MDM2 to regulate p53," Nature Genetics, vol. 29, no. 1, pp. 92-95, 2001.

[43] I. Ringshausen, C. C. O’Shea, A. J. Finch, L. B. Swigart, and G. I. Evan, "Mdm2 is critically and continuously required to suppress lethal p53 activity in vivo," Cancer Cell, vol. 10, no. 6, pp. 501-514, 2006.

[44] F. D. Mairinger, R. F. H. Walter, S. Ting et al., "Mdm2 protein expression is strongly associated with survival in malignant pleural mesothelioma," Future Oncology, vol. 10, no. 6, pp. 9951005, 2014.

[45] P. Kanellou, A. Zaravinos, M. Zioga, and D. A. Spandidos, "Deregulation of the tumour suppressor genes p14ARF, p15 INK4b, p16INK4a and p53 in basal cell carcinoma," British Journal of Dermatology, vol. 160, no. 6, pp. 1215-1221, 2009.

[46] D. Chen, N. Kon, M. Li, W. Zhang, J. Qin, and W. Gu, "ARF$\mathrm{BP} 1 /$ mule is a critical mediator of the ARF tumor suppressor," Cell, vol. 121, no. 7, pp. 1071-1083, 2005.

[47] L. Miao, Z. Song, L. Jin, Y. M. Zhu, L. P. Wen, and M. Wu, "ARF antagonizes the ability of Miz-1 to inhibit p53-mediated transactivation," Oncogene, vol. 29, no. 5, pp. 711-722, 2010.

[48] Y. Huang, T. Tyler, N. Saadatmandi, C. Lee, P. Borgstrom, and R. A. Gjerset, "Enhanced tumor suppression by a p14ARF/p53 bicistronic adenovirus through increased $\mathrm{p} 53$ protein translation and stability," Cancer Research, vol. 63, no. 13, pp. 3646$3653,2003$.

[49] J. W. Martin, S. Chilton-MacNeill, M. Koti, A. J. Van Wijnen, J. A. Squire, and M. Zielenska, "Digital expression profiling identifies RUNX2, CDC5L, MDM2, RECQL4, and CDK4 as potential predictive biomarkers for neo-adjuvant chemotherapy response in paediatric osteosarcoma," PLOS ONE, vol. 9, no. 5, Article ID e95843, 2014.

[50] X. L. Xu, Y. Fang, T. C. Lee et al., "Retinoblastoma has properties of a cone precursor tumor and depends upon cone-specific MDM2 signaling," Cell, vol. 137, no. 6, pp. 1018-1031, 2009.

[51] X. L. Xu, H. P. Singh, L. Wang et al., "Rb suppresses human coneprecursor derived retinoblastoma tumours," Nature, vol. 514, no. 7522, pp. 385-388, 2014.

[52] R. F. H. Walter, C. Vollbrecht, R. Werner et al., "microRNAs are differentially regulated between MDM2-positive and negative malignant pleural mesothelioma," Oncotarget, vol. 7, no. 14, pp. 18713-18721, 2016.

[53] R. F. H. Walter, R. Werner, S. Ting et al., "Identification of deregulation of apoptosis and cell cycle in neuroendocrine tumors of the lung via NanoString nCounter expression analysis," Oncotarget, vol. 6, no. 28, pp. 24690-24698, 2015.

[54] S. Shangary and S. Wang, "Small-molecule inhibitors of the MDM2-p53 protein-protein interaction to reactivate p53 function: A novel approach for cancer therapy," Annual Review of Pharmacology and Toxicology, vol. 49, pp. 223-241, 2009.

[55] L. D. Gamble, U. R. Kees, D. A. Tweddle, and J. Lunec, "MYCN sensitizes neuroblastoma to the MDM2-p53 antagonists Nutlin3 and MI-63," Oncogene, vol. 31, no. 6, pp. 752-763, 2012.

[56] H. D. Herce, W. Deng, J. Helma, H. Leonhardt, and M. C. Cardoso, "Visualization and targeted disruption of protein interactions in living cells," Nature Communications, vol. 4, article no. 2660, 2013.
[57] F. Vandermeers, P. Hubert, P. Delvenne et al., "Valproate, in combination with pemetrexed and cisplatin, provides additional efficacy to the treatment of malignant mesothelioma," Clinical Cancer Research, vol. 15, no. 8, pp. 2818-2828, 2009.

[58] S. A. Bustin, V. Benes, J. A. Garson et al., “The MIQE guidelines: minimum information for publication of quantitative real-time PCR experiments," Clinical Chemistry, vol. 55, no. 4, pp. 611-622, 2009.

[59] R. F. H. Walter, F. D. Mairinger, S. Ting et al., "MDM2 is an important prognostic and predictive factor for platinpemetrexed therapy in malignant pleural mesotheliomas and deregulation of P14/ARF (encoded by CDKN2A) seems to contribute to an MDM2-driven inactivation of P53," British Journal of Cancer, vol. 112, no. 5, pp. 883-890, 2015.

[60] F. D. Mairinger, R. F. H. Walter, S. Ting et al., "Mdm2 protein expression is strongly associated with survival in malignant pleural mesothelioma," Future Oncology, vol. 10, no. 6, pp. 9951005, 2014.

[61] R. Voltan, P. Secchiero, B. Ruozi, F. Forni, C. Agostinis, L. Caruso et al., "Nanoparticles engineered with rituximab and loaded with nutlin-3 show promising therapeutic activity in Bleukemic xenografts," Clinical Cancer Research, vol. 19, no. 14, pp. 3871-3880, 2013.

[62] L. T. Vassilev, B. T. Vu, B. Graves et al., "In vivo activation of the p53 pathway by small-molecule antagonists of MDM2," Science, vol. 303, no. 5659, pp. 844-848, 2004.

[63] S. Trino, L. De Luca, I. Laurenzana et al., "P53-MDM2 pathway: Evidences for a new targeted therapeutic approach in B-acute lymphoblastic leukemia," Frontiers in Pharmacology, vol. 7, p. 491, 2016.

[64] W. Du, J. Wu, E. M. Walsh, Y. Zhang, C. Y. Chen, and Z.-X. J. Xiao, "Nutlin-3 affects expression and function of retinoblastoma protein: role of retinoblastoma protein in cellular response to nutlin-3," The Journal of Biological Chemistry, vol. 284, no. 39, pp. 26315-26321, 2009.

[65] T. He, J. Guo, H. Song et al., "Nutlin-3, an Antagonist of MDM2, Enhances the Radiosensitivity of Esophageal Squamous Cancer with Wild-Type p53," Pathology \& Oncology Research, vol. 24, no. 1, pp. 75-81, 2018.

[66] K. Shimazu, Y. Tada, T. Morinaga et al., "Metformin produces growth inhibitory effects in combination with nutlin-3a on malignant mesothelioma through a cross-talk between mTOR and p53 pathways," BMC Cancer, vol. 17, no. 1, p. 309, 2017.

[67] N. A. Laurie, S. L. Donovan, C.-S. Shih et al., "Inactivation of the p53 pathway in retinoblastoma," Nature, vol. 444, no. 7115, pp. 61-66, 2006.

[68] B. Rao, S. Lain, and A. M. Thompson, "P53-Based cyclotherapy: Exploiting the 'guardian of the genome' to protect normal cells from cytotoxic therapy," British Journal of Cancer, vol. 109, no. 12, pp. 2954-2958, 2013.

[69] R. Voltan, P. Secchiero, B. Ruozi et al., "Nanoparticles engineered with rituximab and loaded with nutlin-3 show promising therapeutic activity in B-leukemic xenografts," Clinical Cancer Research, vol. 19, no. 14, pp. 3871-3880, 2013.

[70] I. Ray-Coquard, J.-Y. Blay, A. Italiano et al., "Effect of the MDM2 antagonist RG7112 on the P53 pathway in patients with MDM2-amplified, well-differentiated or dedifferentiated liposarcoma: an exploratory proof-of-mechanism study," The Lancet Oncology, vol. 13, no. 11, pp. 1133-1140, 2012. 


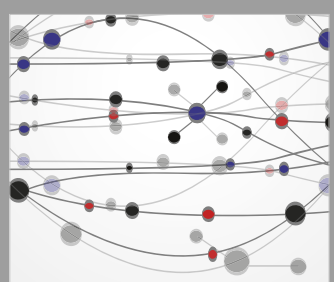

The Scientific World Journal
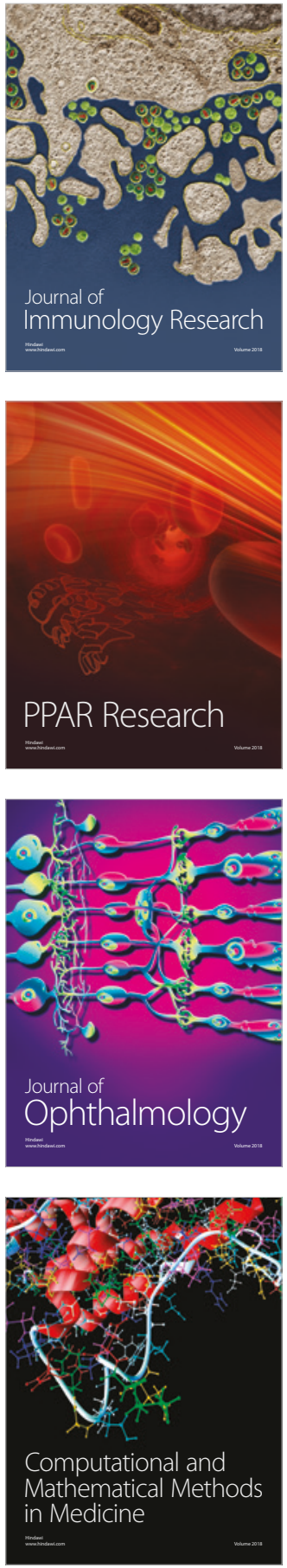

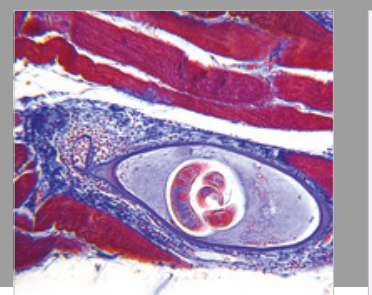

Gastroenterology Research and Practice

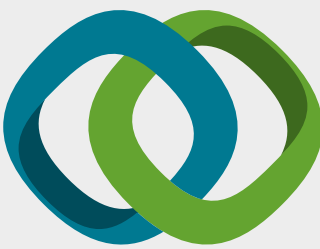

\section{Hindawi}

Submit your manuscripts at

www.hindawi.com
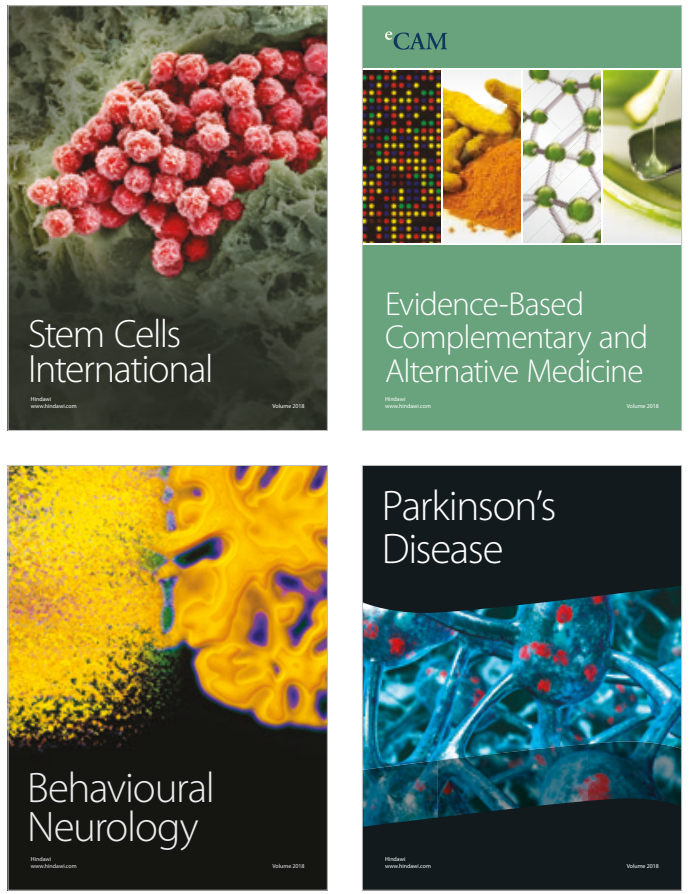

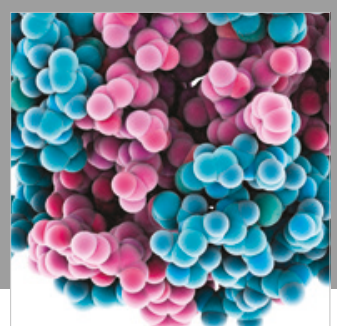

ournal of

Diabetes Research

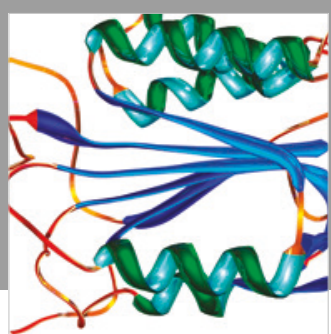

Disease Markers
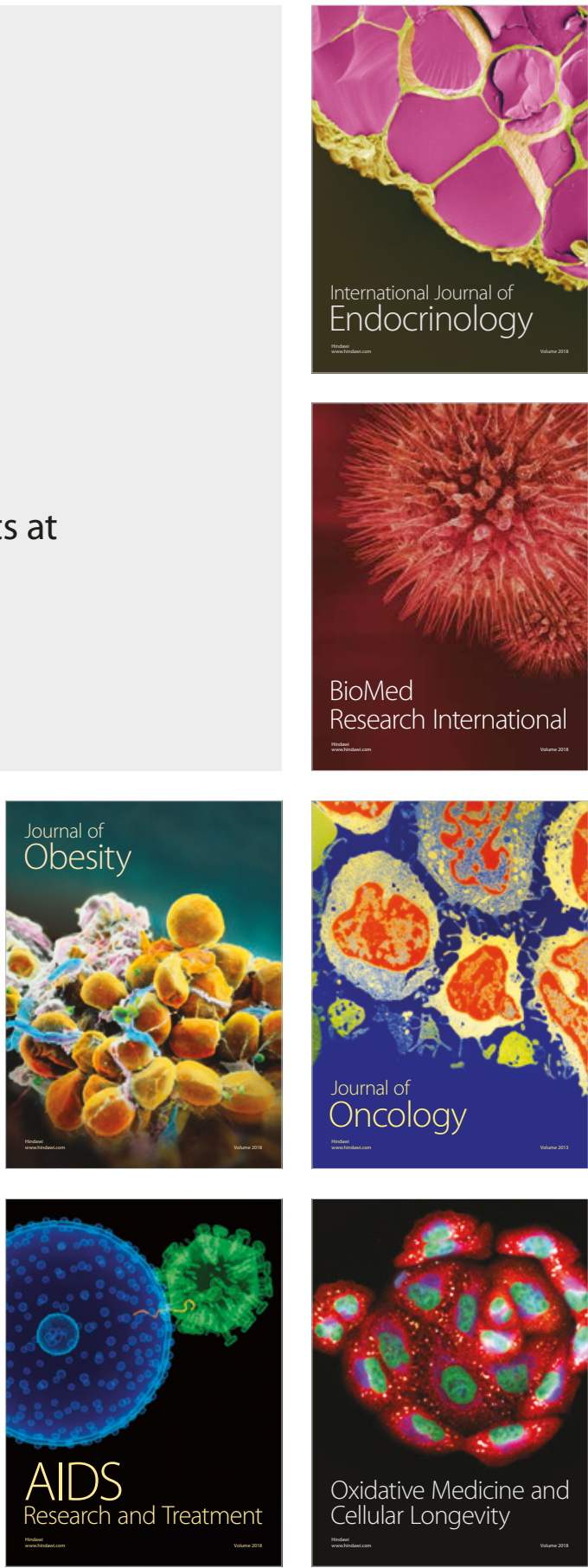